\title{
THE HETEROGENEITY OF SIJRFACES OF MAGNETIC AP STARS
}

\author{
M. HACK \\ Ossematorio Astronomico \\ Trieste, Italy
}

\begin{abstract}
The observations of spectrum-variability and light-variability of Ap stars are reviewed. It is shown that these variations are interpretable as due to the changing aspect of the spotted surface as the star rotates. It is stressed that we understand fairly well the geometry of the phenomenon but the physics is very far from being understood.
\end{abstract}

\section{Introduction}

Magnetic Ap stars are probably those where the presence of a spotted surface is very evident. Their spectrum-variability (profiles, lineintenslty and radial velocity), light-variability and magnetic field variability, all occurring with the same period, are explained in a simple way if we assume that these variations are due to the changing aspect of the spotted surface as the star rotates. The oblique rotator model was proposed by Babcock in 1949 and by Stibbs in 1950 and was worked out in great detail by Deutsch (1954). This model allows us to explain the magnetic field variation from some +1000 to some - 1000 gauss in a few days; it explains the crossover effect, the line-width versus period relations, the line - intensity and radial velocity variation, and in part also the light curves. The main objection against the oblique rotator hypothesis was the supposed existence of many irregularly variable magnetic stars. However, the large number of observations accumulated in the last twenty years indicates that probably all magnetic Ap spectrum-variables are regular variables with periods which are generally of a few days, but includes a small group of long period variables (100 days up to 23 years for HD 9996). The light variability, which is the quantity measurable with the highest precision, has often remained undetected, because the amplitude is always small, in many cases few hundreths of magnitude.

Strong support to the oblique rotator theory is given by the relation $\mathrm{v}$ sin $i$ versus $P$ (Preston, 1971), where $P$ is the period of variability. This relation indicates that all Ap stars fall on or below the curve rotational velocity $\underline{v}$ versus rotational period $P$, where

Edith A. Müller (ed.), Highlights of Astronomy, Vol. 4, Part II, 389-394. All Rights Reserved. Copyright (C) 1977 by the IAU. 
$V=2 \pi R / P$ and $P$ is assumed to be equal to the period of variability, and none falls above it. Thus it is possible to compute $\underline{i}$ from the relation

$$
\sin i=(v \sin i) /(2 \pi R / P)
$$

where $\mathrm{v} \sin i$ is measured from the line broadening, $R$ is given by the relation $L=4 \pi R^{2} \sigma T_{e}^{4}$ and $P$ is the period of spectral or light variability. The knowledge of $i$ allows us to construct models of the spotted surface which are able to reproduce the observations. It is very important to map the surface spots and derive their physical parameters in order to ascertain in which degree the determination of the surface chemical composition is affected. The Nn-stars are not included in these considerations; though they show several abundance peculiarities similar to other groups of Ap stars, none of them has been found variable with certainty.

\section{Spectrum-Variability}

The complex variable profile of several spectral lines indicates that each line is split into several components having different intensity and radial velocity, each of them representing a different spot. A general behavior is the phase relation between velocity and intensity variation, with $W \lambda$ reaching its maximum value when the $R V$ is zero (relatively to the velocity of the stellar center of mass). This means that when the spot crosses the center of the disk its $W \lambda$ is maximum and the corresponding RV is zero; when the spot approaches the limb, projection effects and limb darkening produce a diminution of $W \lambda$. This correlation between line - intensity and RV variation was not always evident in the old works, because the low spectral resolution did not permit the separation of the different components. The importance of using high resolution spectrograms in studying these problems must be stressed here.

A rough determination of the latitude of the spots can be made through the RV curve of each line component. The RV of a spot on the parallel of latitude $\beta$ varies from $-v \sin i \cos \beta$ to $+v \sin i \cos \beta$. Hence the amplitude of the sinusoidal curve $v \sin i \cos \beta \sin \phi$ where $\varnothing$ is the phase $\left(\varnothing=270^{\circ}\right.$ at the approaching limb, $\varnothing=0$ at the center of the disk, $\varnothing=90^{\circ}$ at the receding limb) permits the determination of $\beta$. Moreover, the visibility of a spot will last longer if it is on the hemisphere where the pole is visible. An exemplary application is given by the observations of HD 133029 by Aslanov (1975).

Generally certain groups of elements are concentrated in the same spots, and other elements are diffused over the whole surface or concentrated in other spots, but we cannot recognize a general behavior in the separation of the elements. For instance in some cases iron peak elements present a different distribution than rare earths, in other cases they present the same behavior; sometimes members of the iron peak behave like some rare earths or heavy elements, and other members of the iron peak behave differently. This intriguing behavior 
was observed by Deutsch in 1947; more detailed observations during the past thirty years have not been able to clarify the situation.

Two methods for mapping the distribution of spots on the stellar surface have been employed: a mathematical one developed by Deutsch (1957, 1970), consisting of a harmonic analysis of the spectrum, and an empirical one develped by Khokhlova (1975). Applications of these two methods have been made by Pyper (1969), by Rice (1970), by Megessier. (1974, 1975), and by several researchers of Shemakha Astrophysical Observatory, Azerbaidzhan, USSR, and of the Zentral Institut der Deutschen Akademie der Wissenschaften, DDR, who have started an extensive and systematic research program making use of spectroscopic and photoelectric observations (Aslanov et al.,1973; Ryabchkova, 1974; Glagolevskii et al., 1974).

\section{Light Curves and Spectrum-Variability}

The light variability always occurs with the same period as spectrum and magnetic field variability. Generally maximum light (in $V$ or in UBV system) is in phase with maximum intensity of Eu and other rare earth lines. In hot $\mathrm{Bp}$, generally, $\mathrm{V}$ is in antiphase with He line intensity and in phase with si line intensity. We cannot say if this is a general behavior because no sufficient data are available. From the existing observations it follows that rare-earth spots are general.1y hotter than the photospheric background while He spots seem to be cooler than the photospheric background. This fact, at least in the case of rare earths, can be explained with the blanketing in the far ultraviolet due to the crowding of a large number of lines of rare earth ions. This blanketing produces a backwarming effect in the visible part of the spectrum, where the number of rare earth lines is less numerous, as shown by the OAO-2 observations of several Ap stars (Molnar, 1973, 1975, Leckrone, 1974).

As an example of the importance that mapping the surface of Ap stars can have on empirical tests of the theories proposed to explain theirabundance peculiarities, let us consider the case of HD 193722 (Aslanov et al., 1973). The UBV light curves are in phase with the Eu II lines and this can be explained by blanketing in the ultraviolet and backwarming in the visible. Spots of Si II (Si-rich) are located on a normal background because silicon is strongly in excess. In the same spots there is also a He-concentration, (He-rich), located on a He-deficient background, because the total intensity of helium lines indicates strong He-deficiency. Hence, at least in this star, we have He and $\mathrm{Si}$ excess in the same spots. According to the diffusion theory (Michaud, 1970) we expect, instead, He-deficiency and Si-excess in the same spot. We must consider the possibility that the temperature may be different in the spot from that in the surrounding photosphere and can affect the determination of the abundance.

Several researchers have systematically observed light curves of Ap spectrum-variables (see, for instance, Provin, 1953a, 1953b; Rakos, 1962a, 1962b, 1963; Stepien, 1968a, 1968b, 1968c; Wolff and 
Wolff, 1970, 1971). The majority of these observations have been made in the UBV system. A complete review of the photometric properties of the Ap stars was made by Preston in 1971. The results are confusing because no general behavior can be identified. After this review came the beautiful results from $O A O-2$ ultraviolet observations proving that bianketing and backwarming effects, already suspected by Peterson (1970) and by Wolff and Wolff (1971), are the main reason for the variation of amplitude and shape of the light curve with wavelength. Now the recent series of 10-color photometric observations (from $3400 \mathrm{~A}$ to $7600 \mathrm{~A}$ ) of light curves made by Schöneich et al. (1976 a, $1976 \mathrm{~b}$ ) and by Schöneich and Staude (1976) relative to $16 \mathrm{Ap}$ stars, show that each star is a singular case: an enormous variety of light curves is observed. However, a general behavior can be recognized: the amplitude decreases suddenly at the Balmer limit and rises again in the red. According to these authors, backwarming is not sufficient to explain these curves. The presence of various spots with different concentrations of elements producing different effects of blanketing and backwarming, and possibly a different atmospheric structure in the spots, due to the presence of the magnetic field, might explain the observations. But different combinations of the many variables of the problem can reproduce the observations: it is difficult or impossible to find a unique solution.

\section{Conclusion}

The geometric explanation of spectrum-variability of magnetic stars is very satisfactory; that of the light curves is less satisfactory, although ultraviolet observations have given an important contribution for explaining the phenomenon. However, we do not understand the physics of the problem. Why some elements should concentrate in some spots and othersin other spots? And, if this behavior is correlated with the magnetic properties of the elements, why do they not always follow the same behavior, and are they not always associated in the same group? How stable are the spots? Observations repeated at a distance of months or years agree well generally, suggesting that the life of these spots is much longer than that of the solar spots. However, the existing observations made at a distance of several tens of years very often disagree. It is not clear if the disagreement is entirely real or partly due to the different spectral resolution, which in the old times was too low to allow us to resolve the various components of the spectral lines.

We do not know how the magnetic field affects the atmospheric structure within the spots. Our attempts to understand the phenomena characterizing the magnetic Ap stars is often based on the solar model, however we must recall that Ap stars have general fields of hundreds or thousands of gauss against the solar one, which is less than one gauss: and that an Ap star has little or no convection in the subphotospheric regions while the sun has a thick convective zone. 
We still need high quality observations consisting of high resolution spectrograms, magnetic field measurements, light curves in several colors, from far ultraviolet to infrared, and to repeat these observations for several years at least for a few typical magnetic stars. On the other hand, we need theoretical work in order to understand the behavior of the various elements in the presence of the magnetic field and its effect on the atmospheric structure.

\section{References}

ASLANOV I.A., HILDEBRANDT G., KHOKHLOVA V. L., and SCHONEICH W: 1973 Astrophys. Space Sci. 21,477 ASLANOV I.A.:

1975 Pis'ma Astron.Zh. 1,39 (Sov. Astron.Lett.1,64)

BABCOCK H. W. :

1949 Observatory 69,191

DEUTSCH A. J.:

1947 Astroph.J. 105,283

1954 Trans. IAU $\underline{8}, 801$

1957 IAU Symp. 6,209

1970 Astroph.J. 159,985

GLAGOLEVSKII YU.V., KOZLOVA K.I., and POIOSUKHINA N.S.:

1974 Astrofizika 10,517 (Astrophysics 10,327)

KHOKHLOVA V. L.:

1975 Astron.Zh. 22,950 (Sor.Astron. 19,576)

LECKRONE D.S. :

1974 Astroph.J. 190, 319

MEGESSIER C. :

1974 Astron, Astroph. 34,53

1975 Astron, Astroph. $\underline{39}, 263$

MICHALUD G.:

1970 Astroph.J. 160,641

MOLNAR M.R.:

1973 Astroph.J. 179,527

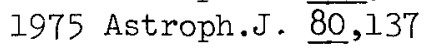

PETEF.SON D.M.:

1970 Astroph.J. 161, 685

PRESTON G. W. :

1971 Publ.Astron.Soc. Pacific 83,571

PROVIN S.S. :

1953 a) Astroph.J. 117,21

1953 b) Astroph.J. 118,489

PYPER D. M. :

1969 Astroph. J.Suppl. 18,347

RAKOSCH K.D.:

1962 a) Lowell Bull. 5,227

1962 b) Z. Astrophys. 56,153

1963

Lowell Bull. $6, \overline{9} 1$ 
RICE J. B. :

1970 Astron. Astroph. 9,189

RYABCHIKOVA T.A.:

1974 Astron.7h. 51,761 (Sov.Astron.18,451)

SCHONEICH W., HILDEBRANDT: $G$, and FURTIG W.:

1976 a) Astron. Nachr. 297,39

SCHONEICH W., KRIVOSHEINA A.A., KHOKHLOVA V.L., and ASLANOV I.A.

1976 b) Astron. Nachr. 297,207

SCFONEICH W., and STAUDE J.:

1976 in press

STEPIEN K.:

1968 a)Astron.J. 73 , S 36

1.968 b) Astroph.J. 153, 165

1968 c) Astroph.J. 154,945

STIBBS I.W.N.

1950 Monthly Notices Roy. Astron. Soc. 110,395

WOLFF, S.C. and WOLFF R. J.:

1970 Astroph. J. 160,1049

1971. Astron.J. 16,422 